\title{
Signs of Life Detection Using Wireless Passive Radar
}

Qingchao Chen, Kevin Chetty

Department of Security and Crime Science

University College London London, U.K

qingchao.chen.13@ucl.ac.uk k.chetty@ucl.ac.uk
Karl Woodbridge

Department of Electronic and Electrical Engineering

University College London London, U.K

k.woodbridge@ucl.ac.uk
Bo Tan

Department of Electronic and Electrical Engineering

University of Bristol, Bristol, UK

b.tan@bristol.ac.uk

\begin{abstract}
Non-contact devices for monitoring signs of life have attracted a lot of attention in recent years for applications in security, emergency and disaster situations. Current devices however, generally utilize bespoke active systems to transmit large bandwidth signals. In this paper, a real-time phase extraction method based on passive Wi-Fi radar is proposed for detecting the chest movements associated with a person breathing. Since the monitored movements are of low amplitude and small Doppler shift, this method uses the phase variation rather than traditional range-Doppler processing. The processing is based on time domain cross correlation, with the addition of a Hampel filter for outlier detection and removal. In this paper the basic passive Wi-Fi model and limitations of traditional cross ambiguity function for signs of life detection are first introduced. The phase extraction method is then described followed by experimental results and analysis. Detection of breathing for a stationary person is shown in both in-room and through wall scenarios using both the Wi-Fi beacon and data transmissions. This is believed to be the first demonstration of signs of life detection using phase extraction in passive radar and extends the capability of such systems into a wide range of new applications.
\end{abstract}

Keywords-Passive Wi-Fi Radar;Breathing Detection; Phase Extraction ; Beacon Signal Detection; Real Time; Through-Wall

\section{INTRODUCTION}

Passive radar systems make use of signals of opportunity such as GSM [1] Wi-Fi [2, 3] and DAB/DVB-T [4] to detect and track targets of interest. They essentially consist of a series of synchronized radio receivers which compare the echoes from moving targets to the original unaltered transmission signal. The receive-only nature of passive radar systems means that they are low-cost, covert, and operate license-free. Moreover, Doppler resolution in passive radar systems can be orders of magnitude better that their counterpart active systems because of the increased integration times possible [5]. Leveraging the increasingly ubiquitous nature of $\mathrm{Wi}-\mathrm{Fi}$ networks, a lot of researches are now being carried out on passive wireless based sensing for a range of applications using cross-correlation processing or other methods such as the use of Channel State Information (CSI). Indoor localization using Doppler-only passive Wi-Fi radar has been demonstrated to localize and track single target [6]. In addition, real time human motion detection has also been investigated using passive $\mathrm{Wi}$ $\mathrm{Fi}$ radar in [3]. The self-transmitted orthogonal frequency division multiplexing (OFDM) signal and modified Fast Fourier Transform (FFT) have been used to extract microDoppler information in [7]while the angle of arrival of targets using self-transmitted OFDM signals and inverse synthetic aperture radar (ISAR) techniques have also been reported in
[8]. Using CSI for motion recognition and localization has been reported in [9] and [10] by interpreting the channel state pattern and fingerprint technology.

Detection of signs of life using wireless or other low bandwidth signal is challenging due to the very small and relatively slow movements involved. Most techniques in this category are therefore based on high bandwidth bespoke active systems and may require complex antenna arrays or the use of wearable devices. WiZ utilizes antenna arrays and a custom wide bandwidth frequency modulated continuous wave (FMCW) signal to measure the time of arrival and relative range to detect breathing for both Line of Sight (LoS) and through-wall scenarios in [11]. Jian et.al. [12]proposed an ultrawideband (UWB) radar to detect breathing. They showed that with the use of a short duration pulse, the range resolution can be small enough to eliminate the reflections from other targets. Dei et.al. [13]demonstrated continuous wave radar centered at frequency of $2.42 \mathrm{GHz}$ for clinical breathing detection, which is simpler but also requires array antennas and clutter suppression hardware. Tan et.al. [14] also reported the performance of breathing detection using a CW radar system in an indoor environment.

In the passive Wi-Fi radar domain, to the author's knowledge, no research has been published to date on breathing detection using passive Wi-Fi systems. In addition, no experimental work has been reported on the use of wireless beacon signal only for signs of life detection. In this paper, we propose and demonstrate a phase extraction method for breathing detection, based on cross correlation processing and Hampel filtering. Experimental detection results from both LoS and through-wall scenarios are analyzed and discussed. We also compare the use of beacon and data burst signals for detection to demonstrate capability in user-quiet periods.

\section{PASSIVE WIFI MODEL FOR BREATHING DETECTION}

In this section, the breathing detection method using passive Wi-Fi radar is described and developed. The passive radar signal model and the cross ambiguity function (CAF) are first introduced and the limitations of normal CAF processing for detecting slow and micro movement are described. The novel phase extraction model based on cross correlation only is then described. The key problems concerning phase sample outliers and the background phase interference are then discussed and Hampel and mean average filters are applied to address these two problems respectively. 


\section{A. Passive Wi-Fi Radar Signal Model and Cross Ambiguity Function Processing}

Passive $W i-F i$ radar utilizes the existing $W i-F i$ access points (APs) as transmitters of opportunity. The reference signal $r e f(t)$, can be described as a linear combination of the transmitted $W i-F i$ signal $x_{\text {source }}(t)$ and the reflections from static objects, characterized by $p^{\text {th }}$ path delay $\tau_{p}$ and the corresponding magnitude and static phase $A_{p}$ :

$$
\operatorname{ref}(t)=\sum_{p} A_{p} x_{\text {source }}\left(t-\tau_{p}\right)
$$

Similarly, the signal in the surveillance channel $\operatorname{sur}(t)$, is composed of echoes from all moving targets in the illuminated area of interest, which can be characterized as the $q^{\text {th }}$ path delay $\tau_{q}$, its Doppler shift $f_{d, q}$ and relevant magnitude and phase $A_{q}$ :

$$
\operatorname{sur}(t)=\sum_{q} A_{q} x_{\text {source }}\left(t-\tau_{q}\right) e^{j 2 \pi f_{d, q} t}
$$

It is assumed that the reference and surveillance signals are separated via the spatially directional antennas.

In general, a target can be identified by cross correlating the reference and surveillance signals and using the Fast Fourier Transform (FFT) to find the exact delay $\tau$ and frequency shift $f$ of the reflected signal. This can be represented by the CAF as follows [2]:

$$
C A F(\tau, f)=\int_{-\infty}^{+\infty} e^{-j 2 \pi f t} r e f^{H}(t-\tau) \times \operatorname{sur}(t) d t
$$

, where the $H$ is the Hermitan operator.

Here, it is worth noting that we treat the Wi-Fi signal $x_{\text {source }}(t)$ as an unknown signal from the Wi-Fi AP and we use the high-gain narrow beam width antennas to try and isolate the $\operatorname{ref}(t)$ and $\operatorname{sur}(t)$ signals in the receivers.

\section{B. Limitations of CAF in Breathing Detection and the Phase Extraction Method}

In normal human breathing, the chest moves slowly and the amplitude of the movement is small. For a passive radar system, detecting breathing is therefore limited by the following factors: firstly, due to the limited bandwidth of the signal, the range resolution of the Wi-Fi radar is around 17 meters which is too coarse for this application; secondly, as the chest movement is slow, a long integration time is required to be able to get fine enough Doppler resolution and this low Doppler could well be masked by zero Doppler clutter or the Direct Signal Interference (DSI).

Here, we analyze breathing detection in more detail and denote the chest movement amplitude as $d(t)$. Accordingly, the phase of the received echo signal from the chest, $\varphi(t)$ can be represented as the following:

$$
\varphi(t)=\frac{2 \pi \times d(t)}{\lambda} .
$$

Due to the limited range resolution of the $\mathrm{Wi}-\mathrm{Fi}$ passive radar, we can therefore assume that the target is in one range bin during each CAF operation. Then the correlation result $x[\mathrm{~m}]$, $m \in[0, M-1]$ in the time domain within a range bin of largest power, $l_{\max }$ is given by:

$$
x[m]=\sum_{n=0}^{N_{m}-1} \operatorname{ref}^{H}\left[i_{m}+n-l_{\max }\right] \times \operatorname{sur}\left[i_{m}+n\right]
$$

where $M$ is the number of batches we divide the signal into and $H$ is the Hermitan operator. Here, the range index $l_{\max }$ represents the range bin with the largest power on the zero Doppler line, $N_{m}$ is the number of data samples in $m^{\text {th }}$ batch and $i_{m}$ is the starting sample index of each batch [3]. The phase of $m^{\text {th }}$ batch can be obtained easily by the following:

$$
\varphi[m]=\arctan \left(\frac{\operatorname{real}(x[m])}{\operatorname{imag}(x[m])}\right)
$$

where the function $\arctan ()$ is the inverse tangent function, the functions real() and imag() exact the real part and imaginary part of the complex number respectively. In the indoor environment, as there are echoes from other reflectors as well, the phase of $x[\mathrm{~m}]$ could be represented as:

$$
\varphi[m]=\frac{2 \pi \times d[m]}{\lambda}+\varphi_{\text {static }}
$$

where $\varphi_{\text {static }}$ is the combination of echo phases from static reflectors, $d[\mathrm{~m}]$ is the relative movement between the chest and the antenna at the $m^{\text {th }}$ batch. Since the slow breathing process, $\varphi[m]$ cannot be larger than half the wavelength and $\varphi_{\text {static }}$ is time-invariant between two consecutive CAF operations, we are able to simultaneously monitor the phase variation corresponding to the chest movement, without considering the distortion from DSI and the long integration time involved in FFT operation.

\section{Outliers in Phase Extraction and the Hampel Filter for Outlier Removal}

Due to modulation characteristics of the Wi-Fi signal, extracting stable phase information from cross correlation is a challenge. The case is even worse when the beacon signals are transmitting due to their lower power and repetition rate. When these less continuous signals are being transmitted, or $x_{\text {source }}(t)$ is sparse in the time domain, there may be many discontinuous phase samples following correlation, as shown in Fig. 2. The reason is that if less data samples are transmitted, the integration power of the echoes will be lower since less reflected data can be utilized for cross correlation. In this way, noisy phase information will provide relatively larger distortions.

To tackle this problem, a Hampel filter is utilized as a postprocessing step to eliminate the outliers [15]. Suppose we have obtained a time series of phase samples, denoted as:

$$
\phi_{k}=\left[\varphi_{k-\omega}, \varphi_{k-\omega+1}, \ldots, \varphi_{k+\omega+1}, \varphi_{k+\omega}\right]
$$

then we would like to check whether $\varphi_{\mathrm{k}}$ is a outlier. Within a certain window size, $\omega$, the median absolute deviation (MAD) scale estimate is calculated as follows:

$$
M A D=\operatorname{median}\left\{\left|\phi_{k}-\operatorname{median}\left\{\phi_{k}\right\}\right|\right\}
$$

Then, with the assumption that within the window, the phase data are sampled according to the following distribution: 


$$
\phi_{k} \sim N\left(\mu_{k}, \sigma_{k}\right)
$$

the estimated deviation of the distribution, $\sigma_{k}$ can be approximately given by the criterion from [16]:

$$
\sigma_{k}=1.4826 \times M A D
$$

where 1.4826 is the calculated in the Gaussian distributions [16]. For a threshold $t$, we could classify the phase as an outlier if $\left|\varphi_{k}\right|>t \times \sigma_{k}$. In this case, the outlier will be set as the median of the series of phase samples.

\section{Mean Average Filter to Eliminate the Static Reflector Inteference}

Although outliers can be eliminated by above methods, phase shifts caused by the background static reflections still remain. Here, due to the time invariant characteristics during consecutive CAF operation, we can use mean phase subtraction for background phase elimination, represented by the following equation:

$$
\phi_{\text {true }, k}=\phi_{k}-\frac{1}{L} \sum_{k-L+1}^{k} \phi_{k}
$$

where $\phi_{\mathrm{k}}$ is the $\mathrm{k}^{\text {th }}$ slow-time sequence of phase information, $L$ is the window length and $\phi_{\text {true }, k}$ is the phase sequence after mean elimination.

\section{IMPLEMENTATION AND EXPERIMENT RESULTS}

In this section, the system design and the implementation of the real-time phase extraction method are described. Experiments in four scenarios are described, including indoor and through-wall scenarios with both beacon and data burst signals. Ranges of results are shown and analyzed.

\section{A. System Design and Implementation}

The Wi-Fi Passive Radar used in these experiments utilizes a Software Defined Radio (SDR) architecture. In this system, two Universal Software Radio Peripheral (USRP) N210s synchronized with a MIMO cable are used to down-convert the RF Wi-Fi signals centered at $2.462 \mathrm{GHz}$. An FPGA (Xilinx Spartan 3A-DSP 3400) and a 100Ms/s, 14-bit ADC are used to digitize the signal. The data are recorded and transferred into a laptop via gigabit Ethernet port for real time processing. The reference and surveillance channel antennas used in the indoor experiments are both small PCB antennas, with 5 dbi gain and 60 degree beam-width. For the through-wall experiments, we used two 15 dbi gain Yagi antennas, with a beam-width of 30 degree. For the Wi-Fi AP, we used an Edimax N300, with two omni-directional antennas of 3 dbi gain.

In the case of both beacon and data signals, we downsampled at $2 \mathrm{MHz}$ and used a 0.5 second integration time with 0.4 second overlapping time between cross correlation operations. We used batch processing with a pipeline flow in Labview for real-time implementation [3]. For detection based on beacon signals, only 10 batches are used while for the one based on data signal, 50 batches are used. It is worth mentioning that the beacon rate is $20 \mathrm{~Hz}$ in our AP setting.

\section{B. LoS Breathing Detection using Beacon and Data Wi-Fi signals}

The indoor experimental scenario is shown in Fig. 1. There are four test positions for breathing (P1 to $\mathrm{P} 4$ ) to allow investigation of different geometries. The vertical distance from the Wi-Fi AP to P1 is $2.5 \mathrm{~m}$ while to the other three it is around $1.5 \mathrm{~m}$. For the surveillance receiver, its vertical distance to P1 is $2 \mathrm{~m}$ while to the others it is around $1 \mathrm{~m}$. During the experiments a person sat on a chair in each position breathing normally.

Fig. 2 shows the phase extraction result for breathing detection using beacon signals only. It can clearly be seen that the phase angle increases during the chest inhaling while the phase angle decreases during exhaling. It can also be seen that approximately 8 to 9 cycles' of breathing are detected within around 40 seconds for all testing positions which corresponds to normal breathing rates. However, although the targets are breathing normally, the magnitudes of the phase variations from the four positions are different. In addition, it can be seen that even for the same position, the phase variations vary slightly from one breathing cycle to the next. Here, a number of probable reasons for these variations are described in the following. Firstly, the wavelength of Wi-Fi signal is around 12 $\mathrm{cm}$ so even slight changes in the geometry due to body movement will change the phase variation. For example, from Fig. 2 (a) and (b), the difference between phase deviations is approximately $0.2 \mathrm{rad}$, which equates to $0.2 \mathrm{~cm}$ difference in chest movement. Secondly, different parts of the chest may be detected in different target positions which may alter the phase deviation somewhat. Finally, although the targets are breathing normally, the chest movement is unlikely to be absolutely regular from one cycle to the next and bulk body movements will cause larger immediate phase changes, such as the red curves in Fig. 2(a) and Fig. 3(c).

Fig. 3 shows the same experiment using data signals. In general, the results are similar to those found using the beacon signals. However, it seems that the phase variations are smoother when using data transmissions as illustrated by comparing Fig 2(a) and 3(a). The main likely reason for this has been discussed in section 2.C. Another potential reason is that since in the case of Beacon signals we need to use longer times for cross correlation, it is more likely that reflected signals from both inhaling and exhaling process will be cross correlated, leading to the phase distortions.

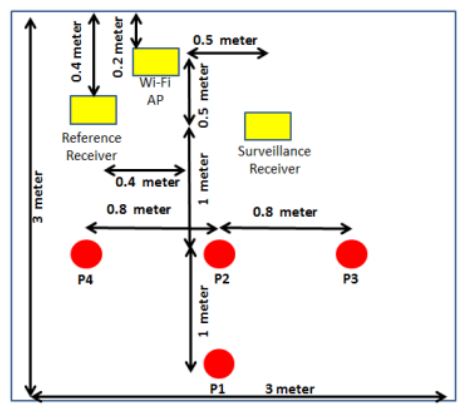

Fig. 1. Indoor Experiment Scenario 

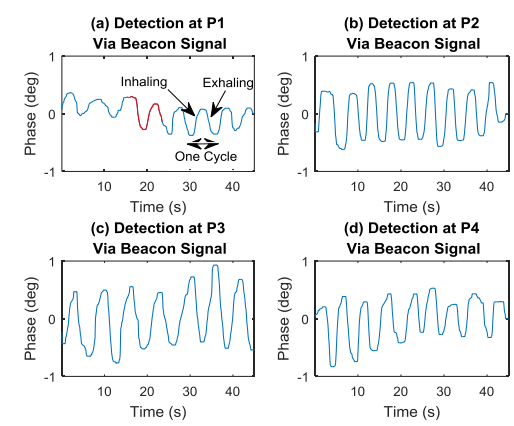

Fig. 2. Breathing Detection Using Beacon Signals on Four Positions
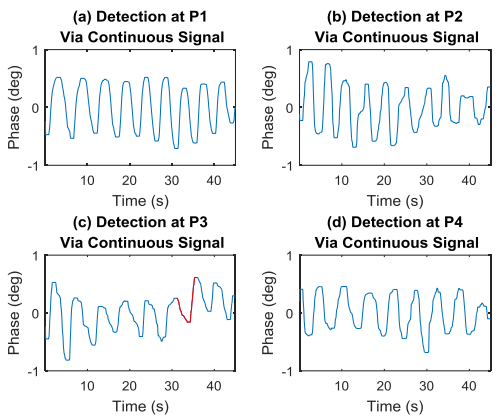

Fig. 3. Breathing Detection Using Continuous Signals on Four Positions

\section{Through-Wall Breathing Detection using Wi-Fi Signals}

The through-wall scenario is shown in Fig. 4. A house with a standard brick/block cavity wall was used (33 cm thickness). The detection zone inside the house was approximately $1.2 \mathrm{~m}$ by $5.4 \mathrm{~m}$ inside the house and a continuously transmitted data Wi-Fi signal was used. Both the reference and surveillance channel antennas are placed outside the house, with $10 \mathrm{~cm}$ and $40 \mathrm{~cm}$ distances from the wall respectively. The Wi-Fi AP was located inside the room, at a height of 1.15 meter and two test positions ( $\mathrm{P} 1$ and $\mathrm{P} 2$ ) are located in the room at a range of 61 $\mathrm{cm}$ and $101 \mathrm{~cm}$ respectively from the wall. It is worth noting that both P1 and P2 were horizontally adjusted to be at approximately on the bore sight of the surveillance and reference antennas. During these experiments, the target was stationary and breathing normally.

Detection results using data burst signals are shown in Fig. 5. The phase variation patterns are similar to those seen in Fig. 2 and 3 and the breathing pattern can still clearly be seen. However, it seems that when target is at $\mathrm{P} 2$, the phase variation pattern is not as stable as the one at P1. This is likely to be because of the weaker signal strength caused by the longer distance of P2. In addition, any small bulk body movements at P2 will have a larger effect on system geometry than such movements at $\mathrm{P} 1$.

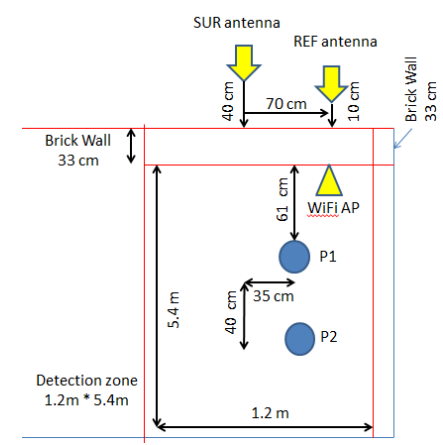

Fig. 4. Through-wall Experiment Scenario

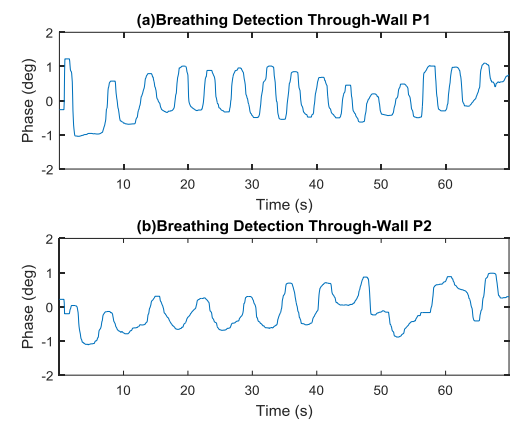

Fig. 5. Breathing Detection Results in Through-wall Scenario

\section{CONCLUSION}

In this paper, a phase extraction method is proposed to detect slow moving and small body movement for signs of life detection using passive Wi-Fi radar. We have extended the traditional cross ambiguity Function processing to include a real-time phase extraction method and Hampel and mean average filter to enhance the detection performance. We demonstrate the effectiveness of this method by experimental demonstration of breathing detection in both line-of-sight and through-wall scenarios. We have been able to detect breathing detection using both Beacon and data Wi-Fi transmissions. This is believed to be the first report of signs of life detection using phase information only in passive Wi-Fi radar. Later on, to investigate more details of the chest movement due to the breathing, the comparison between breathing detection in passive Wi-Fi radar and the active radar system is a good approach.

Passive radar breathing detection using this method has a wide range of applications. These include security and disaster scenarios for detection of concealed or trapped persons and Ehealthcare as a simple and non-contact way to monitor breathing. Finally, it is also worth noting that this phase extraction method is not limited to breathing detection but can also be applied to detection of other small movements such as finger or hand gestures. 


\section{REFERENCES}

[1] D. K. P. Tan, H. Sun, Y. Lu, M. Lesturgie, and H. L. Chan, "Passive radar using Global System for Mobile communication signal: theory, implementation and measurements," Radar, Sonar and Navigation, IEE Proceedings -, vol. 152, pp. 116-123, 2005.

[2] K. Chetty, G. E. Smith, and K. Woodbridge, "Through-the-Wall Sensing of Personnel Using Passive Bistatic WiFi Radar at Standoff Distances," IEEE Transactions on Geoscience and Remote Sensing, vol. 50, pp. 1218-1226, 2012.

[3] B. Tan, K. Woodbridge, and K. Chetty, "A Real-Time High Resolution Passive WiFi Doppler Radar and its Applications," presented at the International Radar Conference, France, 2014.

[4] H. A. Harms, L. M. Davis, and J. Palmer, "Understanding the signal structure in DVB-T signals for passive radar detection," in Radar Conference, 2010 IEEE, 2010, pp. 532-537.

[5] P. E. Howland, "Target tracking using televisionbased bistatic radar," Radar, Sonar and Navigation, IEE Proceedings -, vol. 146, pp. 166-174, 1999.

[6] C. Qingchao, T. Bo, K. Woodbridge, and K. Chetty, "Indoor target tracking using high doppler resolution passive Wi-Fi radar," in Acoustics, Speech and Signal Processing (ICASSP), 2015 IEEE International Conference on, 2015, pp. $5565-5569$

[7] Q. Pu, S. Gupta, S. Gollakota, and S. Patel, "Wholehome gesture recognition using wireless signals," presented at the Proceedings of the 19th annual international conference on Mobile computing \&\#38; networking, Miami, Florida, USA, 2013.

[8] F. Adib and D. Katabi, "See through walls with WiFi!," presented at the Proceedings of the ACM SIGCOMM 2013 conference on SIGCOMM, Hong Kong, China, 2013.
[9] H. Abdelnasser, K. A. Harras, and M. Youssef, "WiGest demo: A ubiquitous WiFi-based gesture recognition system," in Computer Communications Workshops (INFOCOM WKSHPS), 2015 IEEE Conference on, 2015, pp. 17-18.

[10] M. Kotaru, K. Joshi, D. Bharadia, and S. Katti, "SpotFi: Decimeter Level Localization Using WiFi," presented at the Proceedings of the 2015 ACM Conference on Special Interest Group on Data Communication, London, United Kingdom, 2015.

[11] F. Adib, H. Mao, Z. Kabelac, D. Katabi, and R. C. Miller, "Smart Homes that Monitor Breathing and Heart Rate," presented at the Proceedings of the 33rd Annual ACM Conference on Human Factors in Computing Systems, Seoul, Republic of Korea, 2015.

[12] J. Qiuchi, Y. Jian, Y. Yinan, P. Bjorkholm, and T. McKelvey, "Detection of breathing and heartbeat by using a simple UWB radar system," in Antennas and Propagation (EuCAP), 2014 8th European Conference on, 2014, pp. 3078 3081.

[13] D. Dei, G. Grazzini, G. Luzi, M. Pieraccini, C. Atzeni, S. Boncinelli, et al., "Non-Contact Detection of Breathing Using a Microwave Sensor," Sensors, vol. 9, p. 2574, 2009.

[14] T. Bo, A. Burrows, R. Piechocki, I. Craddock, C. Qingchao, K. Woodbridge, et al., "Wi-Fi based passive human motion sensing for in-home healthcare applications," in Internet of Things (WF-IoT), 2015 IEEE 2nd World Forum on, 2015, pp. 609-614.

[15] R. K. Pearson, "Outliers in process modeling and identification," IEEE Transactions on Control Systems Technology, vol. 10, pp. 55-63, 2002.

[16] D. C. Hoaglin, F. Mosteller, and J. W. Tukey, Understanding robust and exploratory data analysis vol. 3: Wiley New York, 1983. 